DOI: $10.18027 / 2224-5057-2021-11-3 s 2-31$

Цитирование: Носов Д.А., Б.Я. Алексеев, Гладков О.А., Волкова М.И., Попов А.М., Харкевич Г.Ю. Практические рекомендации по лекарственному лечению почечноклеточного рака. Злокачественные опухоли : Практические рекомендации RUSSCO \#3s2, 2021 (том 11). 31

\title{
ПРАКТИЧЕСКИЕ РЕКОМЕНДАЦИИ ПО ЛЕКАРСТВЕННОМУ ЛЕЧЕНИЮ ПОЧЕЧНОКЛЕТОЧНОГО РАКА
}

Коллектив авторов: Носов Д.А., Б.Я. Алексеев, Гладков О.А., Волкова М.И., Попов А.М., Харкевич Г.Ю.

Ключевые слова: почечноклеточный рак, иммунотерапия, ингибиторы m-TOR, ингибиторы VEGFR, циторедуктивная нефрэктомия.

\section{1. КЛАССИФИКАЦИЯ И ОПРЕДЕЛЕНИЕ СТАДИИ}

Стадирование почечноклеточного рака (ПКР) должно проводиться по системе TNM (2017 г.) (табл. 1).

Таблица 1. Стадирование рака почки по системе TNM (2017 г.)

\begin{tabular}{|c|c|}
\hline Клиническая стадия & Характеристика \\
\hline \multicolumn{2}{|c|}{ Первичная опухоль (категория Т) } \\
\hline cTx & Первичная опухоль не может быть оценена \\
\hline ст0 & Нет признаков наличия первичной опухоли \\
\hline cT1 & Опухоль $\leq 7$ см в наибольшем измерении, не выходит за пределы почки \\
\hline cT1a & Опухоль $\leq 4$ см в наибольшем измерении, не выходит за пределы почки \\
\hline cT1b & Опухоль > 4 см, но < 7 см в наибольшем измерении \\
\hline cT2 & Опухоль $\geq 7$ см, в наибольшем измерении, не выходит за пределы почки \\
\hline cT2a & Опухоль > 7 см, но $\leq 10$ см, не выходит за пределы почки \\
\hline cT2b & Опухоль > 10 см, но не выходит за пределы почки \\
\hline cT3 & $\begin{array}{l}\text { Опухоль распространяется в крупные вены или периферические ткани, } \\
\text { но не распространяется за пределы фасции Герота и не прорастает ипсилате- } \\
\text { ральный надпочечник }\end{array}$ \\
\hline сТ3а & $\begin{array}{l}\text { Опухоль макроскопически распространяется на почечную вену или её } \\
\text { сегментарные вены (с мышечной стенкой), либо опухоль прорастает в пери- } \\
\text { ренальные ткани и/или почечный синус (в клетчатку, окружающую почечную } \\
\text { лоханку), но не выходит за пределы фасции Герота }\end{array}$ \\
\hline
\end{tabular}




\begin{tabular}{|c|c|}
\hline Клиническая стадия & Характеристика \\
\hline cT3b & $\begin{array}{l}\text { Опухоль макроскопически распространяется в нижнюю полую вену ниже } \\
\text { уровня диафрагмы; }\end{array}$ \\
\hline cT3c & $\begin{array}{l}\text { Опухоль макроскопически распространяется в нижнюю полую вену выше } \\
\text { уровня диафрагмы или прорастает стенку нижней полой вены }\end{array}$ \\
\hline cT4 & $\begin{array}{l}\text { Опухоль распространяется за пределы фасции Герота (и может прорастать } \\
\text { в ипсилатеральный надпочечник) }\end{array}$ \\
\hline \multicolumn{2}{|c|}{ Лимфатические узлы (категория N) } \\
\hline $\mathrm{cNx}$ & Регионарные лимфатические узлы не могут быть оценены \\
\hline CNO & Отсутствие метастазов в регионарных лимфатических узлах \\
\hline cN1 & Метастазы в регионарных лимфатических узлах \\
\hline \multicolumn{2}{|c|}{ Отдаленные метастазы (категория М) } \\
\hline Mx & Оценить наличие отдаленных метастазов невозможно \\
\hline MO & Отдаленные метастазы отсутствуют \\
\hline M1 & Определяются отдаленные метастазы \\
\hline
\end{tabular}

Таблица 2. Соответствие стадий опухолевого процесса категориям TNM

\begin{tabular}{|l|l|l|l|}
\hline Стадия & Категория T & Категория N & Категория M \\
\hline I & T1 & N0 & M0 \\
\hline II & T2 & N0 & M0 \\
\hline III & T3 & N0 & M0 \\
\hline & T1, T2, T3 & N1 & M0 \\
\hline IV & T4 & N любое & M0 \\
\hline & Т любое & N любое & M1 \\
\hline
\end{tabular}

Гистологическая классификация выделяет следующие варианты рака почки (Моch, H., et al. 2016):

- светлоклеточный почечно-клеточный рак;

- мультилокулярная кистозная опухоль с низким злокачественным потенциалом;

- папиллярный почечно-клеточный рак;

- почечный рак, ассоциированный с наследственным лейомиоматозом и почечно-клеточным раком;

- хромофобноклеточный почечно-клеточный рак;

- почечно-клеточный рак из собирательных трубочек;

- медуллярный почечно-клеточный рак;

- почечно-клеточный рак, связанный с транслокацией Міт;

- почечно-клеточный рак, связанный с дефицитом сукцинатдегидрогеназы;

- муцинозный тубулярный и веретеновидноклеточный рак;

- тубулокистозный почечно-клеточный рак; 
- почечно-клеточный рак, ассоциированный с наследственным поликистозом почек;

- папиллярный светлоклеточный почечно-клеточный рак;

- неклассифицируемый почечно-клеточный рак.

Саркоматоидная дифференцировка не является самостоятельным гистологическим вариантом ПКР, может встречаться при любых морфологических формах рака почки, всегда соответствует G4 по Фурману и ассоциирована с неблагоприятным прогнозом.

\section{2. ДИАГНОСТИКА}

Целью обследования при ПКР является оценка локализации и распространенности процесса. Стандартом диагностики и стадирования ПКР является спиральная КТ забрюшинного пространства, брюшной и грудной полостей с в/в болюсным контрастированием. Больным с соответствующими симптомами выполняется радиоизотопное исследование костей скелета с последующим рентгенологическим, однфотонно-эмисиионно-томографическим (ОФЭТ) или МРТ-контролем зон повышенного накопления РФП. Пациентам с общемозговыми и/или очаговыми неврологическими симптомами показано МРТ головного мозга с контрастным усилением. Диагностическая эффективность ПЭТ-КТ с любыми трейсерами при ПКР неизвестна, метод не рекомендован к применению в рутинной практике ввиду его невысокой чувствительности при данном заболевании.

Морфологическую верификацию первичного образования или отдаленных метастазов выполняют с целью установления гистологического варианта опухоли перед назначением системной противоопухолевой терапии, а также перед применением аблативных методов лечения или динамического наблюдения в отношении больных с небольшими размерами первичного образования. У пациентов с множественными злокачественными новообразованиями с целью дифференциальной диагностики источников диссеминации также необходима верификация.

\section{3. ЛЕЧЕНИЕ}

\section{1. Клинически локализованные и местно-распространенные стадии заболевания (T1-4N0-1M0)}

Основным методом лечения при клинически локализованном и местно-распространенном ПКР (сT1-4N0/+М0) является хирургический. Стандартным подходом при клинически локализованном ПКР (сТ1-2) является резекция почки. Нефрэктомия выполняется пациентам с опухолями сT1-2, локализация и размеры которых делают выполнение органосохраняющего лечения технически невозможным, а также больным ПКР сТ3-4. Лимфодиссекция при клинически негативных лимфоузлах (cNO) не является обязательной. При наличии увеличенных забрюшинных лимфоузлов (cN1) выполняется расширенная лимфодиссекция. Аблативные методы в стандарты лечения ПКР не входят и могут рассматриваться как альтернатива хирургическому лечению наряду с динамическим наблюдением у больных 
с крайне высоким операционным риском, имеющих малые периферически расположенные опухоли почки.

После хирургического лечения 10-летняя выживаемость больных в зависимости от стадии варьирует от $30 \%$ до $85 \%$.

После радикального хирургического лечения вне зависимости от стадии адъювантная лекарственная и/или лТ не проводятся, поскольку не способствуют улучшению общей выживаемости больных.

\section{2. Диссеминированная стадия (M1)}

\subsection{1. Факторы прогноза}

Больные диссеминированным ПКР представляют собой разнородную в прогностическом отношении группу. До 2010 г. для оценки прогноза пациентов, которым проводится терапия ИНФ и/или ИЛ -2, использовалась прогностическая модель Memorial Sloan Kettering Cancer Center (MSKCC). Неблагоприятными прогностическим факторами, негативно влияющими на общую выживаемость больных, являются:

- соматический статус (<70\% по шкале Карновского);

- повышение уровня ЛДГ >1,5 ВГН;

- уровень $\mathrm{Hb}<\mathrm{H} Г \mathrm{H}$;

- уровень скорректированного $\mathrm{Ca}^{2+}$ в сыворотке крови >10 мг/длили >2,5 ммоль/л;

- интервал от первичного установления диагноза ПКР до начала терапии <1 года.

В соответствии с моделью MSKCС все пациенты диссеминированным ПКР подразделяются на 3 группы:

- группа благоприятного прогноза (нет факторов риска, медиана продолжительности жизни 30 мес.);

- группа промежуточного прогноза (1-2 фактора риска, медиана продолжительности жизни 14 мес.),

- группа неблагоприятного прогноза ( $\geq 3$ факторов риска, медиана продолжительности жизни 6 мес.).

В настоящее время для оценки прогноза при проведении системной терапии у ранее не леченых больных в клинической практике используется прогностическая модель IMDC (D. Heng 2010), которая представляет собой дополненную модель MSKCC (табл. 1). 
Таблица 3. Прогностическая модель IMDC и показатели выживаемости у пациентов диссеминированным раком почки, получавших системную терапию (D. Heng, 2010).

\begin{tabular}{|c|c|c|}
\hline \multicolumn{3}{|l|}{ Факторы риска: } \\
\hline \multicolumn{3}{|c|}{$\begin{array}{l}\text { 1. Соматический статус по шкале Карновского }<80 \% \\
\text { 2. Концентрация Са } \mathrm{Ca}^{2+} \text { в сыворотке крови }>2,4 \text { ммоль/л } \\
\text { 3. Уровень Нь }<\text { НГН } \\
\text { 4. Время от диагностирования до начала лекарственной терапии <1 года } \\
\text { 5. Уровень нейтрофилов > ВГН } \\
\text { 6. Число тромбоцитов > ВГН }\end{array}$} \\
\hline Прогноз & $\begin{array}{l}\text { Медиана продолжительности } \\
\text { жизни (мес.) }\end{array}$ & 2-летняя выживаемость \\
\hline $\begin{array}{l}\text { Благоприятный } \\
\text { (нет факторов риска) }\end{array}$ & 43,3 мес. & $75 \%$ \\
\hline $\begin{array}{l}\text { Промежуточный } \\
\text { (1 или } 2 \text { фактора риска) }\end{array}$ & 22,5 мес. & $53 \%$ \\
\hline $\begin{array}{l}\text { Плохой } \\
\text { (3 и более факторов риска) }\end{array}$ & 7,8 мес. & $7 \%$ \\
\hline
\end{tabular}

\subsection{2. Лечение}

Выполнение циторедуктивной нефрэктомии до начала таргетной терапии тирозинкиназными ингибиторами возможно только больным группы благоприятного прогноза (IMDC или MSKCC) с потенциально резектабельной первичной опухолью, способным перенести хирургическое вмешательство. В группах промежуточного и неблагоприятного прогноза циторедуктивная нефрэктомия до начала таргетной терапии не улучшает показатели общей выживаемости и может выполняться только пациентам с наличием не более 3-х факторов риска по шкале IMDC.

Роль циторедуктивной нефрэктомии перед проведением комбинированной терапии (ипилимума6 +ниволумаб или ингибитор тирозинкиназы +анти-PD1/PDL-1 антитело) продолжает оставаться не изученной. Выполнение циторедуктивной нефрэктомии возможно при угрожающих жизни состояниях, связанных с наличием первичной опухоли (гематурия, токсико-анемический синдром и др.).

Радикальное удаление солитарных и единичных метастазов может рассматриваться в качестве возможного лечебного подхода у отдельных больных ПКР с индолентным течением болезни. Адьювантная терапия после радикального хирургического удаления метастазов не улучшает результаты лечения, и ее проведение не показано.

лТ может быть использована с паллиативной целью при наличии болевого синдрома у больных с метастатическим поражением костей.

В настоящее время для клинического использования рекомендованы следующие препараты (табл. 4): 
Таблица 4. Препараты и комбинации, рекомендованные для клинического использования в лечении диссеминированного ПКР

\begin{tabular}{|c|c|c|}
\hline Препарат & Группа & Режим лечения \\
\hline $\begin{array}{l}\text { Сунитиниб } \\
\text { Сунитиниб }^{1}\end{array}$ & $\begin{array}{l}\text { Tирозинкиназный ингибитор } \\
\text { VEGFR-1-3, PDGFR, c-KIT, FLT-3 }\end{array}$ & $\begin{array}{l}50 \text { мг/сут. внутрь 1-4 недели, } 2 \text { недели пере- } \\
\text { рыв50 мг/сут. внутрь 1-2 недели, } 1 \text { недели перерыв }{ }^{1}\end{array}$ \\
\hline Сорафениб & $\begin{array}{l}\text { Tирозинкиназный ингибитор } \\
\text { VEGFR-2, RAF-киназы, PDGFR, } \\
\text { c-KIT }\end{array}$ & 800 мг/сутки внутрь \\
\hline Темсиролимус & Ингибитор m-TOR & 25 мг в/в 1 раз в нед. \\
\hline Эверолимус & Ингибитор m-TOR & Эверолимус 10 мг/сут. внутрь \\
\hline Пазопаниб & $\begin{array}{l}\text { Tирозинкиназный ингибитор } \\
\text { VEGFR-1-3, c-KIT, PDGFR }\end{array}$ & 800 мг/сут. внутрь ежедневно \\
\hline Акситиниб ${ }^{2}$ & $\begin{array}{l}\text { Высокоаффинный и селективный } \\
\text { тирозинкиназный ингибитор } \\
\text { VEGFR-1-3 }\end{array}$ & 5 мг× 2 раза в день внутрь ежедневно \\
\hline Кабозантиниб & $\begin{array}{l}\text { Тирозинкиназный ингибитор } \\
\text { MET, VEGFR 1-3, c-KIT, AXL }\end{array}$ & 60 мг × 1 раз в день внутрь ежедневно \\
\hline $\begin{array}{l}\text { Бевацизумаб+ } \\
\text { ИНФ }\end{array}$ & Анти-VEGF MKA & $\begin{array}{l}\text { Бевацизума6 } 10 \text { мг/кг в/в } 1 \text { раз в } 2 \text { недели + ИНФ } \\
\text { 3-6 млн. Ед. п/к } 3 \text { раза в неделю }\end{array}$ \\
\hline Ниволумаб & $\begin{array}{l}\text { анти-PD-1 моноклональное } \\
\text { антитело }\end{array}$ & $\begin{array}{l}3 \text { мг/кг или } 240 \text { мг в/в кап. каждые } 2 \text { недели } \\
\text { или } 480 \text { мг в/в кап. каждые } 4 \text { недели }\end{array}$ \\
\hline $\begin{array}{l}\text { Ниволумаб+ } \\
\text { ипилимумаб }\end{array}$ & $\begin{array}{l}\text { Комбинация анти-PD-1 и анти- } \\
\text { CTLA моноклональных антител }\end{array}$ & $\begin{array}{l}\text { Ниволумаб } 3 \text { мг/кг + ипилимума6 } 1 \text { мг/кг в/в каж- } \\
\text { дые } 3 \text { недели, } 4 \text { введения; далее через } 3 \text { недели } \\
\text { начинается монотерапия ниволумабом } 3 \text { мг/кг } \\
\text { или } 240 \text { мг в/в каждые } 2 \text { недели; или } 480 \text { мг каждые } \\
4 \text { недели }\end{array}$ \\
\hline $\begin{array}{l}\text { Ленватиниб+ } \\
\text { Эверолимус }\end{array}$ & $\begin{array}{l}\text { Комбинация тирозинкиназного } \\
\text { ингибитора FGFR 1-4, RET, VEGFR } \\
\text { 1-3 и ингибитора m-TOR }\end{array}$ & $\begin{array}{l}\text { Ленватиниб } 18 \text { мг/сутки внутрь + Эверолимус } \\
5 \text { мг/сутки внутрь }\end{array}$ \\
\hline $\begin{array}{l}\text { Пембролизу- } \\
\text { маб + акситиниб }\end{array}$ & $\begin{array}{l}\text { Комбинация анти-PD-1 МКА } \\
\text { с тирозинкиназным ингибитором } \\
\text { VEGFR 1-3 }\end{array}$ & $\begin{array}{l}\text { Пембролизумаб } 200 \text { мг в/в кап., каждые } 3 \text { нед. } \\
\text { в комбинации с акситинибом } 5 \text { мг× } 2 \text { раза в день }\end{array}$ \\
\hline $\begin{array}{l}\text { Пембролизумаб+ } \\
\text { ленватиниб }\end{array}$ & $\begin{array}{l}\text { Комбинация анти-PD-1 МКА } \\
\text { с тирозинкиназным ингибитором } \\
\text { FGFR 1-4, RET, VEGFR 1-3 }\end{array}$ & $\begin{array}{l}\text { Пембролизумаб } 200 \text { мг в/в кап., каждые } 3 \text { нед. } \\
\text { в комбинации с ленватинибом } 20 \text { мг/сут. внутрь }\end{array}$ \\
\hline $\begin{array}{l}\text { Авелумаб+ } \\
\text { акситиниб }\end{array}$ & $\begin{array}{l}\text { Комбинация анти-PD-L1 MKA } \\
\text { с тирозинкиназным ингибитором }\end{array}$ & $\begin{array}{l}\text { Авелумаб } 10 \text { мг/кг в/в кап., каждые } 2 \text { нед. } \\
\text { в комбинации с акситинибом } 5 \text { мг× } 2 \text { раза в день }\end{array}$ \\
\hline $\begin{array}{l}\text { Ниволумаб+ } \\
\text { кабозантиниб }\end{array}$ & $\begin{array}{l}\text { Комбинация анти-PD-1 MКА } \\
\text { с тирозинкиназным ингибитором } \\
\text { MET, VEGFR 1-3, c-KIT, AXL }\end{array}$ & $\begin{array}{l}\text { Ниволумаб } 240 \text { мг в/в кап. каждые } 2 \text { недели, } \\
\text { кабозантиниб } 40 \text { мг× } 1 \text { раз в день внутрь ежедневно }\end{array}$ \\
\hline
\end{tabular}

1 сунитиниб в данном режиме (2/1 нед.) используется только в случае непереносимости стандартного режима (4/2 нед.).

2 Начальная доза акситиниба - 5 мг ×2 раза в день, при отсутствии токсических осложнений IIIIV cmепени в течение первых двух недель приема производится эскалация дозы препарата до 7 мг ×2 раза в день (в течение последующих двух недель) и далее-до 10 мг ×2 раза в день. 


\section{Алгоритм выбора режима лекарственной терапии у больных ПкР}

В первой линии терапии светлоклеточного ПКР (сПКР) группы благоприятного прогноза в зависимости от клинической ситуации предпочтительно проведение терапии комбинациями пембролизумаб +ленватиниб, пембролизумаб +акситиниб, ниволумаб +кабозантиниб или авелумаб +акситиниб. Использование данных комбинаций у пациентов с благоприятным прогнозом не приводит к статистически значимому увеличению общей выживаемости, но сопровождается достоверным увеличением частоты объективных ответов, а также достоверным увеличением (пембролизумаб +ленватиниб, ниволумаб +кабозантиниб или авелумаб +акситиниб) либо тенденцией к увеличению выживаемости без прогрессирования (пембролизумаб +акситиниб, ниволумаб +кабозантиниб). В качестве альтернативных режимов, особенно у пациентов с минимальной опухолевой нагрузкой и/или противопоказаниями к назначению комбинированных режимов, может использоваться пазопаниб или сунитиниб.

В первой линии терапии сПКР групп промежуточного и неблагоприятного прогноза предпочтительно назначение следующих комбинированных режимов: ниволумаб +ипилимумаб, пембролизумаб +акситиниб, пембролизумаб +ленватиниб, или ниволумаб +кабозантиниб. В качестве альтернативного режима с меньшим уровнем доказательности может рассматриваться комбинация авелумаб +акситиниб. Возможной опцией при наличии противопоказаний к проведению иммунотерапии является назначение кабозантиниба в монорежиме. Использование комбинированных режимов ассоциируется с более высокой частотой нежелательных явлений 3-4 ст. и требует тщательного мониторинга за пациентами. При использовании комбинации ниволумаб + ипилилумаб частота иммуноопосредованных нежелательных явлений 3-4 ст. увеличивается до $46 \%$.

Роль сорафениба и комбинации ИНФ +бевацизумаб в зависимости от прогноза пациентов в настоящее время остается малоизученной, также как и роль темсиролимуса в группе неблагоприятного прогноза в сравнении с другими препаратами и режимами в связи с крайне ограниченным числом клинических исследований. Данные препараты в настоящее время не могут рассматриваться в качестве режимов предпочтения или альтернативных режимов.

Больным сПКР, у которых развилась резистентность к антиангиогенной терапии тирозинкиназными ингибиторами в монорежиме или к комбинации ИНФ +бевацизумаб, предпочтительно назначение ниволумаба или кабозантиниба. В качестве альтернативы возможно проведение терапии комбинацией ленватиниба с эверолимусом. У отдельных пациентов, имеющих противопоказания к использованию режимов предпочтения или альтернативного режима, во второй линии допускается назначение акситиниба или эверолимуса. Частота нежелательных явлений 3-4 ст. при использовании кабозантиниба или комбинации ленватиниба с эверолимусом может достигать $70 \%$ и требует тщательного мониторинга переносимости терапии.

У больных сПКР с резистентностью на фоне или после использования комбинированных режимов в первой линии оптимальный лекарственный подход продолжает оставаться предметом клинических исследований. При прогрессировании болезни после назначения иммунотерапевтической комбинации ипилимумаб +ниволумаб возможно использование тирозинкиназныхингибиторов в монорежиме - кабозантиниба, акситиниба, сунитиниба или пазопаниба. В случае прогрессирования болезни на фоне комбинации тирозинкиназ- 
ного ингибитора с анти-PD1/PD-L1 MKA оптимальный выбор терапии для последующей линии остается не изученным. В этих случаях возможной опцией является назначение кабозантиниба или комбинации ленватиниб +эверолимус, если данные препараты не использовались ранее. В качестве допустимых режимов возможно использование также других тирозинкиназных ингибиторов, которые ранее не использовались.

Алгоритм выбора режима лекарственной терапии у больных ПКР представлен в табл. 5 и 6.

Таблица 5. Алгоритм выбора режима лекарственной терапии первой линии у больных сПКР

\begin{tabular}{|l|l|l|l|}
\hline $\begin{array}{l}\text { Предшествующее } \\
\text { лечение }\end{array}$ & $\begin{array}{l}\text { Группа } \\
\text { прогноза IMDC }\end{array}$ & Режимы предпочтения & $\begin{array}{l}\text { Альтернативные } \\
\text { режимы }\end{array}$ \\
\hline Не было & Благоприятный & Пембролизумаб+ ленватиниб & $\begin{array}{l}\text { Пазопаниб }{ }^{1} \\
\text { Сунитиниб }^{1}\end{array}$ \\
\hline & & Пембролизумаб+ акситиниб & \\
\hline & Авелумаб+ акситиниб & \\
\hline & Промежуточный & Ниволумаб+ ипилимумаб & Кабозантиниб \\
\hline & Пембролизумаб+ акситиниб & Авелумаб+ акситиниб \\
\hline & Неблагоприятный & Ниволумаб+ ипилимумаб & \\
\hline & Пембролизумаб+ ленватиниб & Кабозантиниб \\
\hline & & Ниволумаб+кабозантиниб & Авелумаб+ акситиниб \\
\hline & & Пембролизумаб+ ленватиниб & \\
\hline & & Ниволумаб+ кабозантиниб & \\
\hline
\end{tabular}

1 При противопоказаниях к анти-PD1 препаратам

Таблица 6. Алгоритм выбора режима лекарственной терапии второй и последующих линий у больных сПКР

\begin{tabular}{|l|l|l|l|}
\hline Предшествующее лечение & $\begin{array}{l}\text { Режимы } \\
\text { предпочтения }\end{array}$ & $\begin{array}{l}\text { Допустимые режимы } \\
\text { ольтернативные режимы }\end{array}$ \\
\hline $\begin{array}{l}\text { Тирозинкиназные } \\
\text { ингибиторы в монорежиме }\end{array}$ & $\begin{array}{l}\text { Ниволумаб } \\
\text { Кабозантиниб }\end{array}$ & Ленватиниб+ эверолимус & $\begin{array}{l}\text { Акситиниб } \\
\text { Эверолимус }\end{array}$ \\
\hline $\begin{array}{l}\text { Ипилилумаб+ ниволумаб } \\
\text { Клинические } \\
\text { исследования }\end{array}$ & $\begin{array}{l}\text { Клинические исследова- } \\
\text { ния }\end{array}$ & $\begin{array}{l}\text { Кабозантиниб } \\
\text { Акситиниб } \\
\text { Сунитиниб } \\
\text { Пазопаниб }\end{array}$ \\
\hline $\begin{array}{l}\text { Тирозинкиназные } \\
\text { ингибиторы+ } \\
\text { анти-PD1/PDL-1 }\end{array}$ & $\begin{array}{l}\text { Клинические } \\
\text { исследования }\end{array}$ & $\begin{array}{l}\text { Кабозантиниб1 } \\
\text { Ленватиниб1+ эверолимус }\end{array}$ & $\begin{array}{l}\text { Сунитиниб } \\
\text { Пазопаниб } \\
\text { Акситиниб! }\end{array}$ \\
\hline
\end{tabular}

\footnotetext{
1 Если препарат не использовался в первой линии терапии
} 


\section{4. НЕСВЕТЛОКЛЕТОЧНЫЙ РАК ПОЧКИ И РАК ПОЧКИ С САРКОМАТОИДНЫМ КОМПОНЕНТОМ}

Лекарственная терапия несветлоклеточного ПКР (нПКР) не изучалась в рандомизированных исследованиях III фазы. В программах расширенного доступа было показано, что прогноз больных нПКР хуже, чем у пациентов со светлоклеточными опухолями. При папиллярном ПКР (1 и 2 типа) предпочтительной опцией является кабозантиниб. На основании результатов систематического анализа, включившего данные небольших исследований 2 фазы (ESPN, RECORD -3, ASPEN, KEYNOTE -427), сунитиниб, пембролизумаб и, в меньшей степени, эверолимус остаются предпочтительными опциями для лечения пациентов с другими формами нПКР.

При раке собирательных трубочек возможно назначение XT комбинацией цисплатин +гемцитабин или карбоплатин +гемцитабин (при противопоказаниях к назначению цисплатина). При ПКР с наличием саркоматоидного компонента необходимо использовать режимы, включающие анти-PD1/PD-L1 MKA (ипилилума6 +ниволумаб или комбинация тирозинкиназных ингибиторов и анти-PD1/PD-L1 MKA). Желательно включение больных нПКР в клинические исследования.

\section{5. ПОБОЧНЫЕ ЭФФЕКТЫ, СВЯЗАННЫЕ С ПРОВЕДЕНИЕМ ЛЕКАРСТВЕННОГО ЛЕЧЕНИЯ}

Наиболее частыми побочными эффектами, характерными для ингибиторов VEGFR, являются астения, АГ, диарея, ЛПС, гипотиреоз, кожная токсичность, нейтропения. Для бевацизумаба характерными видами токсичности являются протеинурия, угроза кровотечения, слабость. Частота и степень выраженности данных побочных явлений может варьировать в зависимости от использования того или иного препарата. До начала и на фоне проведения терапии тирозинкиназными ингибиторами необходимо осуществлять мониторинг гематологических показателей, АД и функции щитовидной железы (ТТГ, ТЗ, Т4). Перед назначением препарата следует добиться адекватного контроля АД (антагонисты кальция, ингибиторы АПФ или блокаторы рецептора к ангиотензину II, бета-блокаторы) и, при необходимости, продолжить гипотензивную терапию. Развитие АГ и гипотиреоза в течение первых 4-6 недель лечения ассоциируется с более высокими показателями беспрогрессивной и общей выживаемости. Медикаментозная коррекция АД и гипотиреоза, развившихся на фоне таргетной терапии, не влияет на результаты лечения и должна проводиться в соответствии с общепринятыми рекомендациями.

Характерными нежелательными явлениями, ассоциированными с терапией ингибиторами ITTOR (темсиролимус, эверолимус), являются мукозиты, пульмониты, иммуносупрессия и риск инфекции, а также метаболический синдром (гипергликемия, гиперхолестеринемия, гиперлипидемия). До старта терапии ингибиторами ITOR следует оценить и, при необходимости, скорректировать исходные показатели липидов и глюкозы сыворотки крови, излечить персистирующие инфекции, а также исключить клинически значимое 
снижение дыхательной функции. В процессе лечения необходимо мониторировать указанные показатели.

При проведении иммунотерапии ингибиторами контрольных точек (ниволумаб, пембролизумаб, ипилимумаб) в различные сроки могут развиваться аутоиммунные поражения различных систем органов различной степени выраженности. Чаще всего регистрируются проявления кожной и гастроинтестинальной токсичности, реже - аутоиммунные эндокринопатии (аутоиммунный гипофизит, тиреоидит, поражение надпочечников), нефропатии, гепатиты, нейропатии. 